\title{
Network Context Aware based Integrated Multimedia Mobility Service Management Scheme over Wireless Networks
}

\author{
Ronnie D. Caytiles and Byungjoo Park ${ }^{*}$ \\ Department of Multimedia Engineering, Hannam University \\ 133 Ojeong-dong, Daeduk-gu, Daejeon, Korea \\ rdcaytiles@gmail.com, *bjpark@hnu.kr
}

\begin{abstract}
The advent of the rapidly increasing evolution of wireless telecommunication technologies has allowed the vast proliferation of multimedia traffic across the heterogeneous wireless networks. Thus, a robust and seamless mobility support management is required in order for an efficient delivery of multimedia services in this ubiquitous environment. This paper deals with the utilization of the fast proxy mobile Internet protocol version 6 (FPMIPv6) in the analysis of a distributed mobility management (DMM) support to provide a seamless handover between wireless networks. The evolution of DMM has paved a way for the distributed delivery of multimedia services in the optimized manner as compared to the centralized mobility management solutions. It aims to negate the limitations of the centralized mobility management warranting the seamless real-time multimedia services provided by a robust handover performance.
\end{abstract}

Keywords: distributed mobility management, heterogeneous wireless networks, FPMIPv6

\section{Introduction}

Nowadays, the wireless telecommunication technologies are rapidly evolving that goes with the widespread deployment of low-cost local wireless access points. In addition, the exponential and continuous growth of mobile multimedia traffic is expected that requires the deployment of more wireless network infrastructures [1]. The idea of installations is to make the mobile network systems closer to the users in order to increase its efficiency with regards to spectrum and coverage area. This mixture of wireless access networks covers different ranges but are overlapping in the covered geographical areas of the ubiquitous environment. The handheld mobile devices (e.g., smartphones, PDAs, tablets, laptops) are now capable of multitasking and can access multiple multimedia services with different network systems.

With this fast changing ubiquitous environment, operators of network systems are faced with several challenges in delivering an efficient and seamless multimedia service to the users. The mobility management to support the handover for the mobile devices is essentially important for providing seamless and continuous network connectivity across the different wireless networks. Each wireless network technology is required to work together in order that Quality of Service (QoS) multimedia services can be warranted. In this regard, Internet Engineering Task Force (IETF) and the $3^{\text {rd }}$ Generation Partnership Project (3GPP) are working on the standardization of several mobility management solutions. Most of these approaches are considered to be centralized in providing mobility support for mobile devices as

Received (May 25, 2017), Review Result (August 28, 2017), Accepted (September 19, 2017)

* Corresponding Author 
it roams across the heterogeneous wireless networks. That is, centralized mobility anchors (e.g., home agents, local mobility anchor) are responsible for allocating the distribution of multimedia IP packets along with the mobility-related functions. The use of centralized mobility anchors in directing multimedia traffic across heterogeneous wireless networks suffers from several drawbacks such as low scalability, reliability (i.e., single point of failure), sub-optimal routing, higher packet loss rate, signaling overhead, lack of granularity on the mobility management service, and leads to a more complex infrastructure deployment $[2,3]$.

This paper aims to address the limitations that are identified with the centralized mobility management solutions which are essentially important to provide the Internet users their desired QoS multimedia services anytime and anywhere. It deals with the analysis of the fast handovers for Proxy Mobile Internet Protocol version 6 (FPMIPv6) based distributed mobility management (DMM) solution to support the handovers of mobile devices as they roam around different wireless network systems. The DMM is a new flat architectural paradigm designed for the evolving multimedia services wherein the mobility anchors are placed at the edge of the wireless network systems and is closer to the Internet users. It is aimed to provide a seamless and balanced flow of multimedia traffic in the ubiquitous environment where data and control traffic are separated in order to optimize its local distribution.

The rest of this paper is organized as follows: Section 2 provides an overview of mobility management solutions; the analysis of the fast handovers for PMIPv6 based distributed mobility management handover of mobile devices across the heterogeneous wireless networks is discussed in Section 3; and the concluding remarks in Section 4.

\section{Mobility Management Overview}

Heterogeneous wireless networks require interoperability among different access networks as they have their own attributes and application requirements. Their attributes varies in spectrum, accessibility, signaling, and infrastructure. The performance of mobility support can be measured through the perceived quality of service $(\mathrm{QoS})$ delivery of multimedia services to the users. In this regard, there is a need for a mobility management solution that takes the full advantage of IP enabled technologies in order to support an effective handover of network connectivity for mobile devices across different access networks.

The term mobility refers to the ability of a mobile device to roam across access networks without losing its reachability in order for the ongoing sessions to be maintained. The IP mobility can be can be classified into host-based or networkbased mobility management. The mobile device in the host-based mobility management takes part on the mobility-related signaling, whereas, in the networkbased, the mobility-related signaling is relegated to the network entities. The Mobile Internet Protocol version 4 (MIPv4) [4] illustrates the mobility support for mobile nodes (MNs) allowing them to move between network domains and maintain its reachability as proposed by the Internet Engineering Task Force (IETF). The home agent (HA) is responsible for intercepting all IP packets intended to the MN and directs it to the MN's home address (HoA). The MN acquires a temporary Care-of Address (CoA) as it moves to a foreign network that is used to receive the IP packets. The intercepted IP packets in the HA is encapsulated and forwarded to the foreign agent (FA) to where the $\mathrm{MN}$ is currently attached and is responsible for decapsulating the IP packets to be delivered to the MN's CoA. The delivery of IP packets from HA to the FA may take longer routes. The correspondent nodes (CNs) need not to know the movement of the MN, wherein, it continuously sends all IP packets through the HA. 
Due to the rapidly increasing number of mobile users and real-time multimedia services readily available, the MIPv4 remains to be an inadequate solution for the mobility management concerns as it suffers from various limitations such as triangular routing, shortage of addresses, signaling overhead, higher handover latency, high packet loss rate, etc. Thus, the Mobile Internet Protocol version 6 (MIPv6) [5] is standardized in order to address such limitations levied by MIPv4. It has allowed the MN to move within the Internet domain while maintaining its reachability with its CNs. The IP traffic is still intercepted by the HA (which acts as the mobile anchor) and directly forwarded to the MN's CoA instead that it will go through the longer route with the FA. This means that the $\mathrm{CN}$ can reach the MN anytime and anywhere.

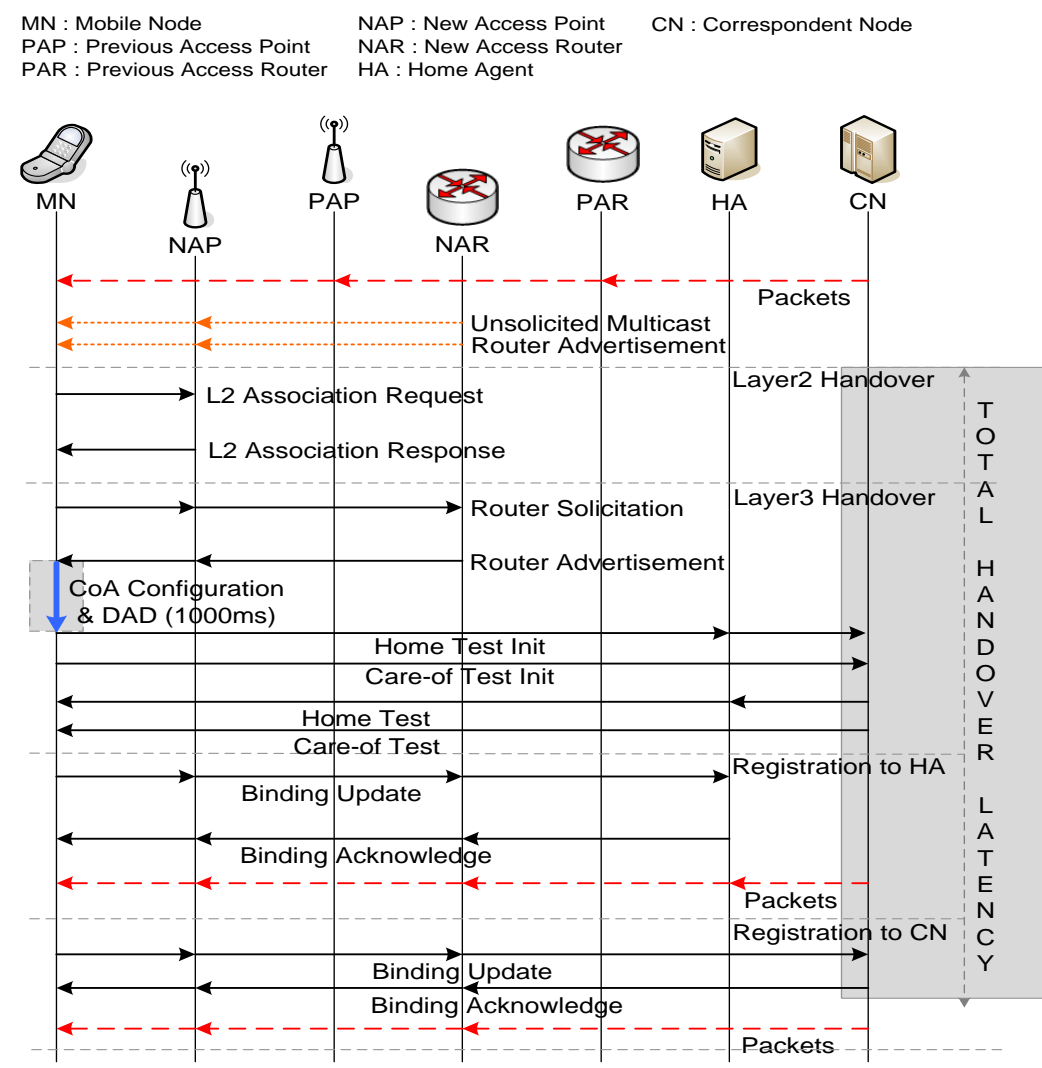

Figure 1. The MIPv6 Handover Process

The handover process for MIPv6 is shown in Figure 1. The MN in MIPv6 is provided with a fixed home address (HoA) and a temporary Care-of Address (CoA) whenever it changes its point of attachment within the Internet domain. Whenever the $\mathrm{MN}$ is within its home network, the HA delivers the IP packets directly to the MN's HoA using the normal routing process. If the MN changes its point of attachment to another network, the intercepted IP packets by the HA is tunneled directly to the current CoA of the MN. Every time the MN moves into another network, it is required to update the HA with its current CoA. MIPv6 optimization has improved the reliability of the network and addressed some limitations of MIPv4; however, it still suffers from numerous drawbacks such as high handover latency, high packet loss rate, and signaling overheads [6].

The Fast Mobile Internet Protocol version 6 (FMIPv6) [7] was introduced in order to address the high handover latency and packet loss issues of MIPv6 to 
provide QoS multimedia services to Internet users with minimized disruptions. The handovers are anticipated using link layer events (L2 triggers) and the IP packets are tunneled to the new access router (NAR) prior to the movement of the MN. The MN then advertises its presence to the NAR and will start receiving the multimedia service IP packets as soon as the $\mathrm{HA}$ and $\mathrm{CN}$ has received the binding update (BU) message.

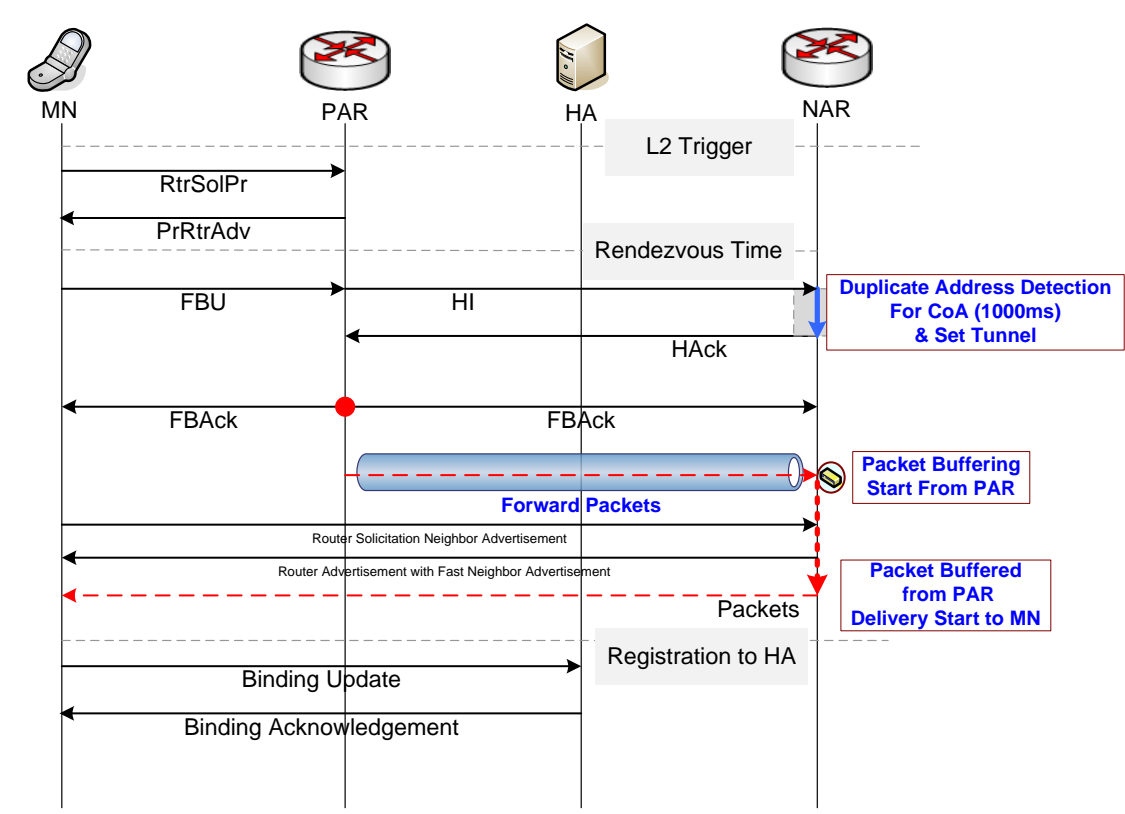

Figure 2. The FMIPv6 Handover Process

The FMIPv6 handover process is shown in Figure 2. The tunneling starts from the previous access router (PAR) ending at the PAR allowing the old CoA of the MN to be used while the handover is still on process. The FMIPv6 is design to make the handover faster in order to reduce the high handover latency and packet loss in the standard MIPv6 but it must always rely on an accurate handover prediction, thus, there are times that it can lead into erroneous handover detection and early L2 triggers.

The Proxy Mobile Internet Protocol version 6 (PMIPv6) is a network-based mobility management protocol that is standardized by the IETF Network-based Localized Management (NTLMM) working group (WG) [8,9]. The MN can move across the heterogeneous wireless networks without being involved in the mobility related signaling as they are relegated to the network entities, thus, the $\mathrm{MN}$ becomes independent of its movement. There are two network entities that are introduced in PMIPv6:

(1) Mobility access gateway (MAG) performs the mobility related signaling operations for the mobile user device that are currently attached into its access points. It is residing in one of the access routers (AR) and is responsible for MN's movement detection, router and neighbor discovery, routing states coordination, IP connectivity provision, and signaling operations.

(2) Local mobility anchor (LMA) maintains the collection of IP address of all MNs attached within the localized mobility domain (LMD). It is residing in the MN's home network and is acting as the local HA. 


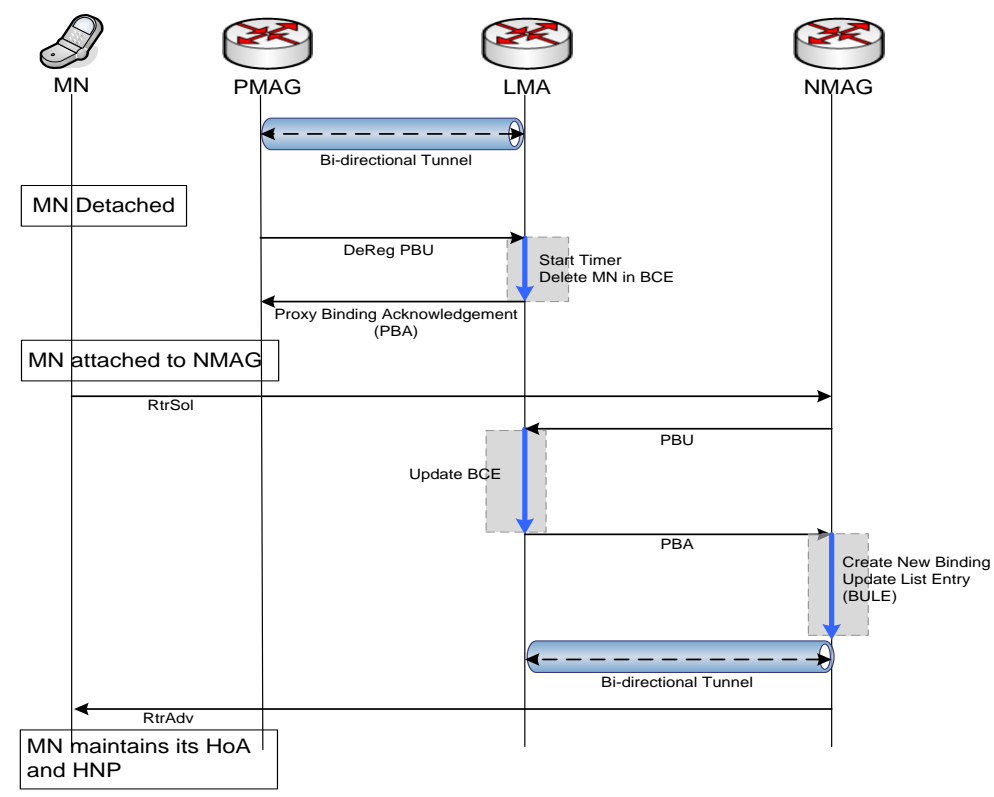

Figure 3. The PMIPv6 Handover Process

The handover process for PMIPv6 is shown in Figure 3. A bidirectional tunnel is created between the LMA and MAG, thus, the MN maintains its originally assigned IP address within the LMD. The LMA intercepts all IP packets that are intended to the MN and tunnels them towards the MAG to which the MN is currently attached. And locally, the MAG decapsulates the received IP packets and forward it to the MN. The PMIPv6 handover is faster as compared to the standard MIPv6 since the former has made the signaling update time shortened, however, link layer handover takes time to be performed resulting to packet losses during this period.

In order to address the issues (e.g., handover latency and packet loss) posed by the PMIPv6, the Fast Proxy Mobile Internet Protocol version 6 (FPMIPv6) [10, 11] is standardized with some extensions for FMIPv6. FPMIPv6 operates in either of the following modes as shown in Figure 4:

(1) The predictive mode, the $\mathrm{MN}$ is the one initiating the handover through sending an indication message to the previous mobile access gateway (PMAG).

(2) The reactive mode, the MN executes re-attachment to the new mobile access gateway (NMAG) and the NMAG will initiate the handover process before the MN can inform the PMAG of the necessary handover. 


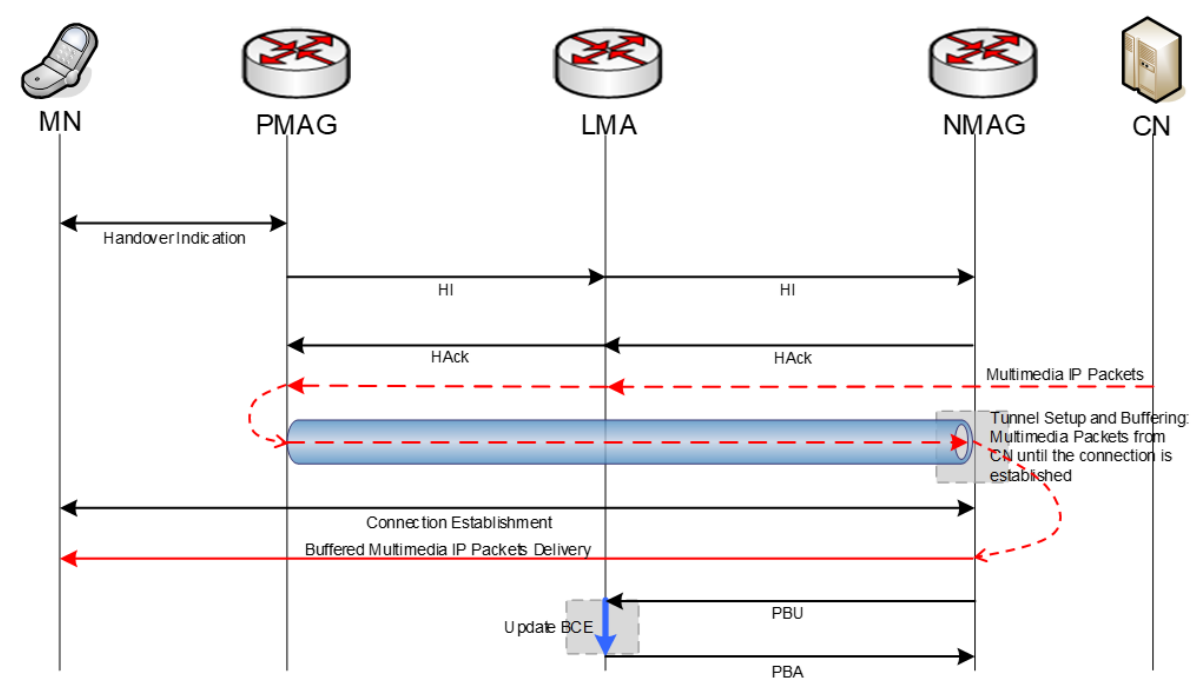

(a) Predictive Mode

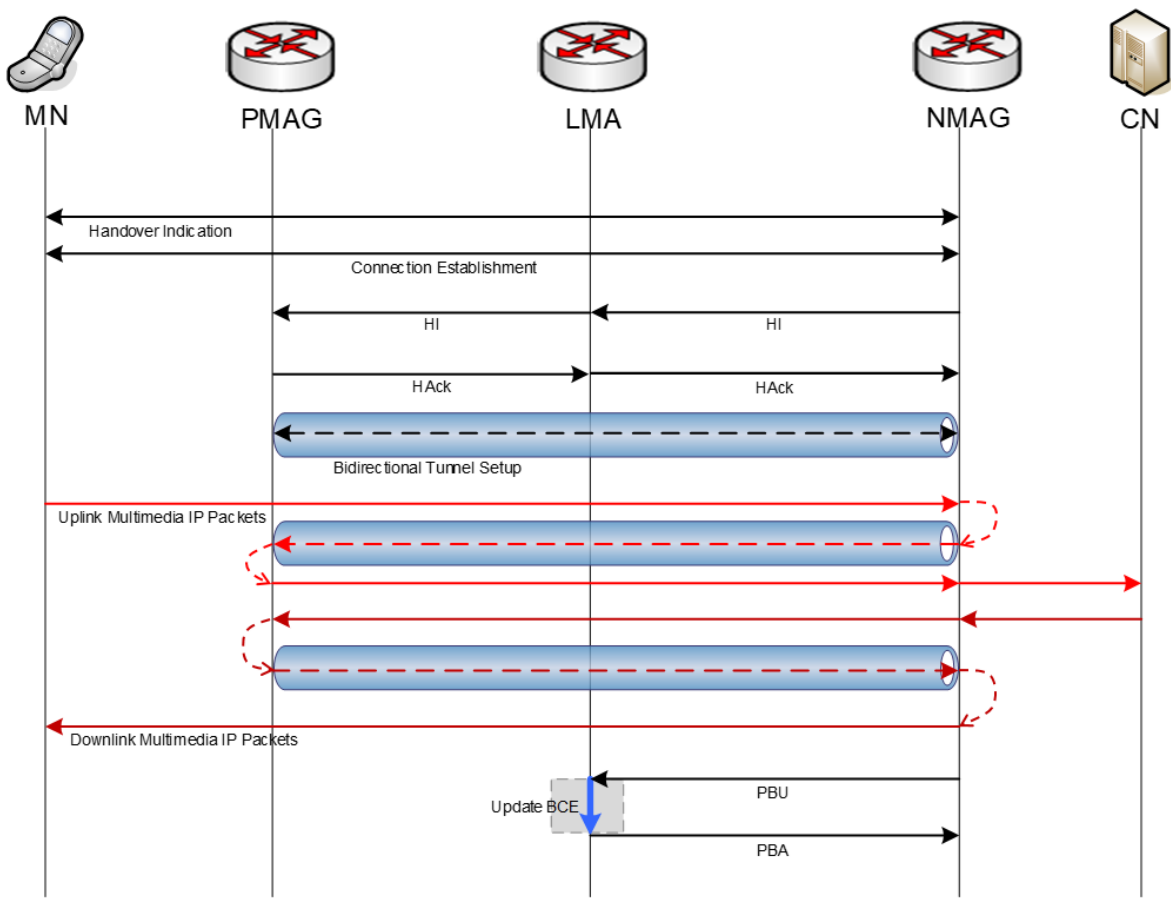

(b) Reactive Mode

Figure 4. The FPMIPv6 Handover Process

The handover delay for PMIPv6 is minimized by the FPMIPv6 since the IP packets are forwarded to and from the MN as the handover is detected. In the predictive mode, the MN in FPMIPv6 is not involved in the mobility-related signaling, but the MN needs to be capable of sending the lower-layer information of handover indication to the PMAG. In the reactive mode, the NMAG sends the HI message to the PMAG after the MN has moved its point of attachment to the NMAG.

\section{FPMIPv6 based DMM Support Analysis}

The optimizations for the MIPv6 are considered to be centralized mobility management solutions for delivering multimedia services to the Internet users employed in hierarchical 
network systems. The role of LMA in PMIPv6 and HA in MIPv6 is considered to be the centralized mobility anchor for the movement of the MN across the heterogeneous wireless networks as well as for the delivery of multimedia services. LMA and HA serve as the central controller for the distribution of data traffic between interconnected wireless networks. The centralized mobility management support is not a fully optimized solution considering the increasing demand for multimedia data traffic across the heterogeneous wireless networks. Thus, the DMM paradigm [12] as a new architectural paradigm is introduced in order to enhance QoS on the delivery of multimedia services to the Internet users.

The DMM is a flat system architecture that places the mobility anchors at the edge of the access networks which are very close to the users. The delivery of the multimedia services is distributed among the mobility anchors to where the MNs are attaching their connectivity. The network-based DMM solutions can be classified into fully distributed and partially distributed schemes. The fully distributed scheme combines the data and control planes as they are managed by the mobility anchors, that is, both the LMA and MAG functions are implemented. While data and control planes are separated in the partially distributed scheme allowing the mobility anchors to distribute only the data plane carrying the multimedia services traffic and all control management is implemented by a particular mobility management entity (MME).

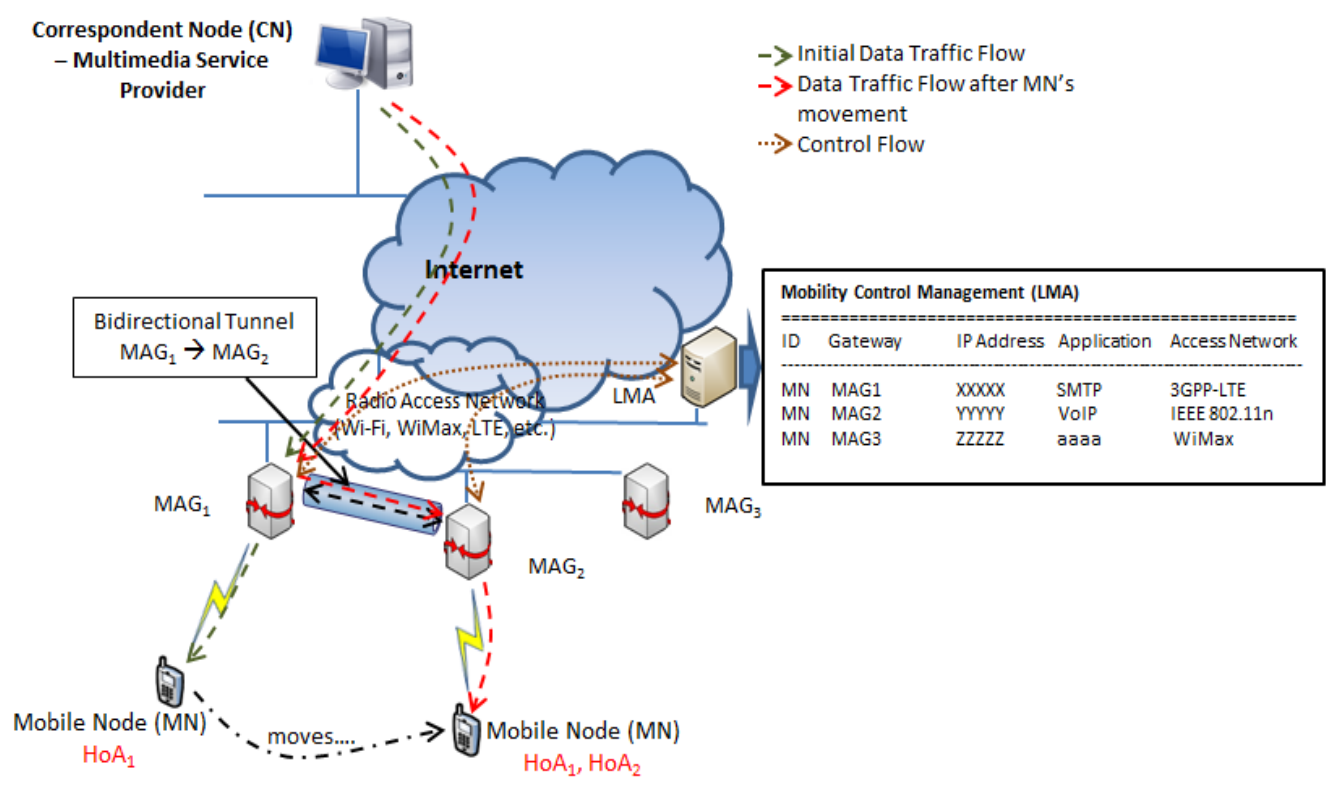

(a) FPMIPv6 based DMM Operations 


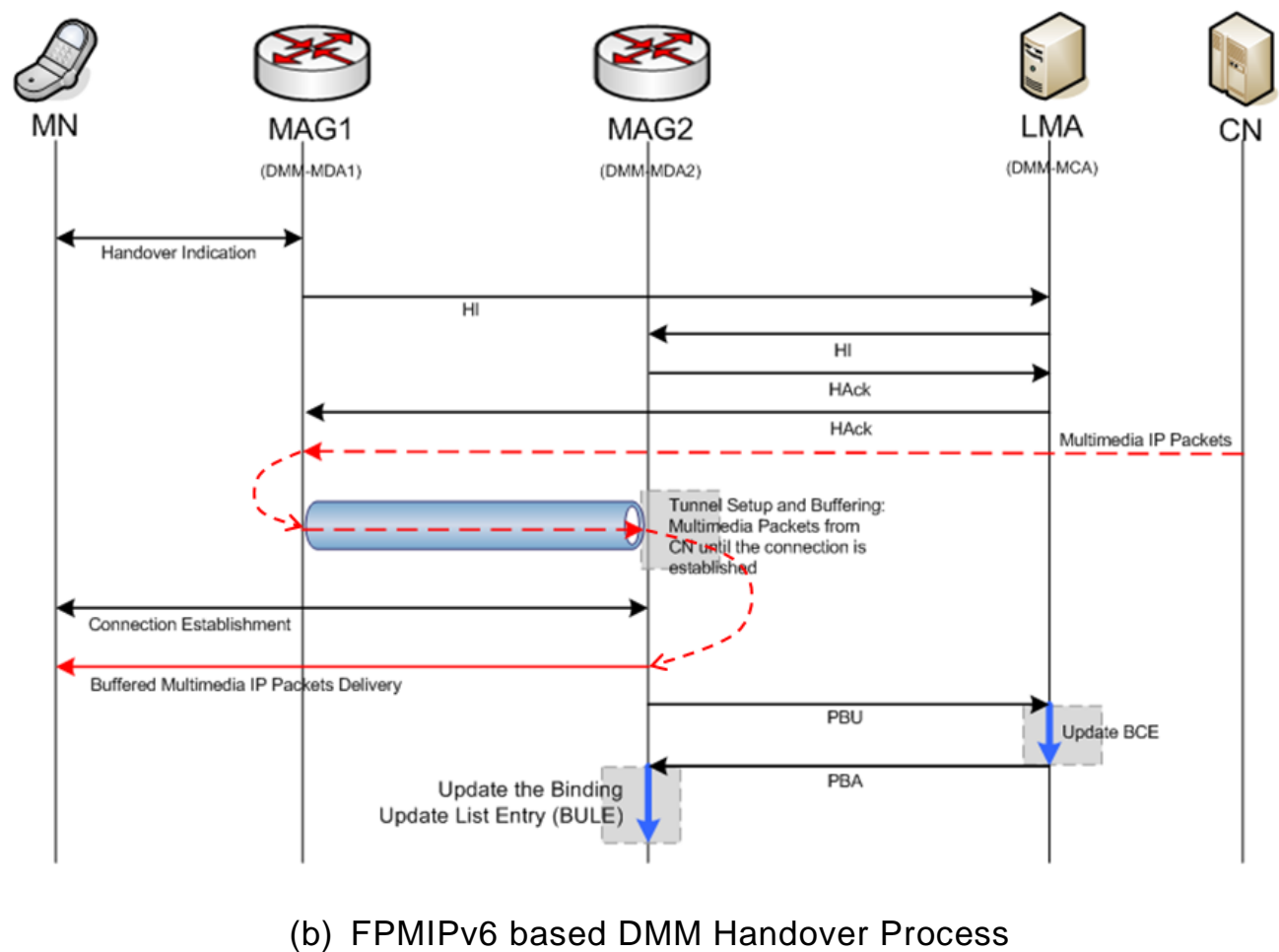

Figure 5. FPMIPv6 based DMM Multimedia Service Delivery

The proposed scheme is based on the predictive mode of fast handovers for PMIPv6 and utilizes the partially distributed scheme of DMM. The multimedia service traffic is locally distributed by the mobile anchors while the control signaling for mobility support is managed by a single centralized entity. The FPMIPv6 based DMM support scheme is depicted in Figure 5 wherein the mobility anchors are implemented by the available MAGs and the centralized entity of DMM is implements the functional operations of the LMA. The mobile devices (MNs) can change its point of attachments between overlapping coverage of MAGs. With the predictive mode of fast handovers, the bidirectional tunnel between MAGs is created before the connection of the MN is established with the new MAG. Thus, multimedia service traffic can be forwarded from the previous MAG prior to the movement of the MN. The multimedia service traffic is buffered in the new MAG until MN's connectivity is established. Then, the multimedia service traffic is decapsulated and forwarded to the MN.

The network access control mechanisms (e.g., mobility management, session management, etc.,) are handled by the LMA which acts as the mobility control anchor for the handover scheme. It maintains the information for directing the flow of multimedia service traffic between MAGs and its forwarding to the corresponding MNs. The MN is no longer involved in the mobility-related signaling except that the MN is required to be capable of reporting the indication of handover. The MAGs implements the functional operations of the mobility data anchors which are responsible for multimedia service traffic distribution to the Internet users. It is responsible for providing the access links to the MNs and is responsible for MN's movement detection, router and neighbor discovery, routing states coordination, IP connectivity provision, and signaling operations to support the mobility of MNs. The mobility-related signaling is only exchanged between the LMA and MAGs.

Moreover, multimedia service traffic no longer passes through the LMA, instead it is directly forwarded to the MAGs. That is, LMA is responsible for managing the control plane while MAGs are allocating the data plane. Thus, multimedia service traffic no 
longer goes back to the network core resulting to a more optimal route towards its distribution to the Internet users.

\section{Conclusion}

The rapidly increasing volume of multimedia traffic available over the Internet has brought the demand for increased bandwidth and a seamless mobility support in order to provide the mobile users with QoS multimedia services. This paper has presented an analysis of FPMIPv6 based DMM multimedia service delivery over the integrated heterogeneous wireless networks. The proposed scheme has analyzed the utilization of the predictive mode of fast handovers for PMIPv6 and integrated with the partially distributed DMM scheme. This result to an efficient distribution of multimedia service traffic to be optimized for the allocation is closer to the Internet users. In addition, it has addressed the limitations of the centralized mobility solutions in the issues of scalability, reliability, packet loss rate, and suboptimal routing.

\section{Acknowledgments}

This research was supported by Basic Science Research Program through the National Research Foundation of Korea (NRF) funded by the Ministry of Science, ICT and future planning (2015R1A2A2A03002851).

\section{References}

[1] CISCO, "Cisco Visual Networking Index: Forecast and Methodology, 2016-2021", White Paper, https://www.cisco.com/c/en/us/solutions/collateral/service-provider/visual-networking-indexvni/complete-white-paper-c11-481360.html, (2017) September.

[2] P. Bertin, S. Bonjour and J. Bonnin, "Distributed of centralized mobility", In Proceedings of IEEE Global Telecommunications Conference (GLOBECOM 2009), IEEE, (2009), pp. 1-6.

[3] H. A. Chan, H. Yokota, J. Xie, P. Seite and D. Liu, "Distributed and Dynamic mobility Management in Mobile Internet: Current Approaches and Issues", Journal of Communications, Academy Publisher, vol. 6, Issue 1, (2011), pp. 4-15.

[4] C. Perkins, "IP Mobility Support for IPv4", Internet Engineering Task Force (IETF), RFC 3344, (2002) August.

[5] C. Perkins, D. Johnson and J. Arkko, "Mobility Support in IPv6", Internet Engineering Task Force (IETF), RFC 6275, ISSN: 2070-1721, (2011) July.

[6] I. Al-Surmi, M. Othman and B. M. Ali, "Mobility Management for IP-Based Next Generation Mobile Networks: Review, Challenge and Perspective", Journal of Network and Computer Applications, vol. 35, no. 1, (2012) January, pp. 295-315.

[7] R. Koodli, "Fast Handovers for Mobile IPv6", Internet Engineering Task Force, RFC 4068, (2005) July.

[8] S. Gundavelli, K. Leung, V. Devarapalli, K. Chowdhury and B. Patil, "Proxy mobile IPv6", Internet Engineering Task Force (IETF), RFC 5213, (2008) August.

[9] C. J. Bernardos, M. Gramaglia, L. M. Contreras, M. Calderon and I. Soto, "Network-based Localized IP mobility Management: Proxy Mobile IPv6 and Current Trends in Standardization", Journal of Wireless Mobile Networks, Ubiquitous Computing, and Dependable Applications (JoWUA) (Special issue: Advances in Wireless Mobile and Sensor Technologies), vol. 1, no. 2/3, (2010), pp. 16-35.

[10] H. Yokota, "Fast Handovers for PMIPv6", Internet Engineering Task Force, RFC 5949, (2010) September.

[11] J.-M. Chung, "Enhancements to FPMIPv6 for Improved Seamless Vertical Handover between LTE and Heterogeneous Access Networks", IEEE Wireless Communications, vol. 20, no. 3, (2013) June, pp. $112-9$.

[12] J. C. Zúñiga, "Distributed Mobility Management: A Standards Landscape", IEEE Communications Magazine, vol. 51, no. 3, (2013) March, pp. 80-87. 
International Journal of Control and Automation

Vol. 10, No. 10 (2017) 